\title{
Review Article \\ Role of Investigating Thrombophilic Disorders in Young Stroke
}

\author{
Kay W. P. Ng, Pei K. Loh, and Vijay K. Sharma \\ Division of Neurology, Department of Medicine, National University Hospital, 1E, Kent Ridge Road, Singapore 119228 \\ Correspondence should be addressed to Vijay K. Sharma, drvijay@singnet.com.sg
}

Received 15 September 2010; Revised 22 December 2010; Accepted 5 January 2011

Academic Editor: Halvor Naess

Copyright ( 12011 Kay W. P. Ng et al. This is an open access article distributed under the Creative Commons Attribution License, which permits unrestricted use, distribution, and reproduction in any medium, provided the original work is properly cited.

\begin{abstract}
Our knowledge about various inherited and acquired causes of thrombophilic disorders has increased significantly during the past decade. Technology for various diagnostic tests for these rare disorders has matched the rapid advances in our understanding about the thrombophilic disorders. Inherited thrombophilic disorders predispose young patients for various venous or arterial thrombotic and thromboembolic episodes. Our understanding has also improved about various gene-gene and gene-environment interactions and their impact on the resultant heterogenous clinical manifestations. We describe various thrombophilic disorders, their diagnostic tests, pathogenic potential in isolation or with other concurrent inherited/acquired defects and possible therapeutic and prophylactic strategies. Better understanding, optimal diagnostic and screening protocols are expected to improve the diagnostic yield and help to reduce morbidity, disability, and mortality in relatively younger patients harbouring these inherited and acquired thrombophilic disorders.
\end{abstract}

\section{Introduction}

Ischemic stroke (IS) is a common cause of morbidity and mortality with significant socioeconomic impact especially when it affects young patients. The incidence of ischemic stroke varies from 3 to 23 per 100,000 among the young IS sufferers [1]. Although the cutoff age for defining young IS remains debatable, it is generally believed that the risk factors and underlying etiologies tend to become similar to the older patients at around 44 years of age. Therefore, most of the studies define "stroke in young" as occurring in patients at age 44 years or less [2]. Young strokes generate immense interest among the stroke neurologists even when a larger proportion of patients continue to be classified as "stroke of undetermined etiology" [2].

Compared to the older adults, the incidence, risk factors, and etiology are distinctly different in younger IS. Accordingly, cardioembolism (20\%-35\%), dissection of extracranial arteries $(6 \%-25 \%)$, drugs $(10 \%)$, and hypercoagulable states $(5 \%-10 \%)$ are relatively more commonly detected in younger IS patients [3-5]. Furthermore, additional factors such as migraine, pregnancy and oral contraceptive use are observed with higher frequency $[4,5]$.

Young IS attracts a barrage of diagnostic tests, mainly searching for an underlying thrombophilic state. We discuss various thrombophilic disorders, their available diagnostic tests and significance of testing for these uncommon causes.

\section{Thrombophilic Disorders and Ischemic Stroke}

Thrombophilic states are disorders of hemostatic mechanisms that result in a predisposition to thrombosis [6]. Thrombophilia is an established cause of venous thrombosis. Therefore, it is tempting to assume that these disorders might have a similar relationship with arterial thrombosis. However, thrombophilia, alone, rarely causes arterial occlusions. Even in individuals with a positive thrombophilia screen and arterial thrombosis, the former might not be the primary etiological factor [7]. Although thrombophilic disorders and their contribution to the stroke risk are uncommon, their detection often helps in management decisions, long-term prognostication, screening family members "at-risk", and possible primary prophylaxis.

Thrombophilic disorders can be broadly divided into inherited or acquired conditions. Inherited thrombophilic disorders are far less commonly observed in young IS. These include deficiencies of natural anticoagulants such as protein C, protein S, and antithrombin III (AT III) deficiency, 
polymorphisms causing resistance to activated protein $\mathrm{C}$ (Factor V Leiden mutation), and disturbance in the clotting balance (prothrombin gene 20210G/A variant). Of all the inherited thrombophilic disorders, Factor V Leiden mutation is perhaps the commonest cause, accounting for about half of the cases. Prothrombin gene mutation, protein C, protein $S$ deficiency, and antithrombin deficiency account for most of the remaining cases. On the contrary, acquired thrombophilic disorders are more common and include conditions such as the antiphospholipid syndrome, associated with lupus anticoagulant and anticardiolipin antibodies.

In general, primary thrombophilic disorders contribute to about $1 \%-4 \%$ of ischemic strokes. However, the prevalence varies widely between different ethnic groups, age, and geographic distributions. An exhaustive 15-years follow-up study of 150 families with different inherited thrombophilic disorders failed to demonstrate any association between these coagulation defects and arterial thrombosis [8]. However, these inherited thrombophilias were associated with a high incidence of venous thrombosis, conferring almost $100 \%$ risk of deep vein thrombosis (DVT) in protein S deficiency. DVT with or without pulmonary embolism was the most frequent type of thrombosis. Similar observations were reported in a case-control study that looked at the association between inherited thrombophilic disorders and acute IS [7]. It found that 1 in 7 patients with firstever acute IS had a positive test for at least one of the thrombophilias. However, this relationship was considered coincidental instead of an actual cause of stroke for all the IS subtypes, even when adjusted for age [6].

\section{Thrombophilic Disorders}

3.1. Factor $V$ Leiden. Although factor V Leiden mutation accounts for almost half of the thrombophilic states, its prevalence varies among different ethnic groups. It is more commonly seen in Caucasians (prevalence $1 \%$ to $8.5 \%$ ). However, Factor V Leiden mutation has been reported rarely among patients of Chinese, Japanese, and African descent $[8,9]$. Although this mutation predisposes to a higher risk of venous thrombosis, its association with arterial disease remains unestablished for myocardial infarction as well as IS [9]. In a study of 203 young IS patients, Nedeltchev et al. reported $0.9 \%$ incidence of Factor V Leiden mutation [10].

3.2. Prothrombin G20210A Mutation. Wide variations are observed in the geographic and ethnic prevalence of prothrombin (factor II) G20210A gene mutation. Similar to the Factor V Leiden, this mutation is also rare in patients of Asian and African descent, but occurs in about $0.7 \%$ to $4.0 \%$ of the Caucasians [11]. Although Prothrombin gene mutation is not considered a risk factor for IS in the older populations, an association has been suggested in the younger population $[12,13]$. In a study of younger IS patients (below ages 50 and 60 years, respectively), without other established vascular risk factors, were more likely to have this gene mutation as compared to the controls (6-7.6 versus $1-1.2 \%)$ [12]. However, another study of 131 patients with IS contradicted these results and found that the prevalence of Prothrombin gene mutation was similar to the control population [13].

\subsection{Deficiencies in Coagulation Factors (Inherited Coagulop-} athies). Inherited coagulopathies are generally more common in Black Africans and Black Caribbeans, with or without IS. In a prospective study of multiethnic population, black African patients were found to have significantly lower protein $\mathrm{S}(P<.001)$ protein $\mathrm{C}(P=.049)$, and antithrombin III $(P=.056)$ levels when compared to the whites [14]. Similar trends were noted for protein $\mathrm{S}$ deficiency in a smaller study that reported its higher incidence in blacks than nonblacks (34\% versus $13 \%, P=.001$ ) [15].

Protein $\mathrm{C}$ is one of the three key proteins that regulate coagulation besides protein $S$ and antithrombin III. It is a vitamin $\mathrm{K}$-dependent glycoprotein and its deficiency presents in 2 forms. Type I deficiency has plasma protein C concentration below $70 \%$ of the overall mean value. Type II deficiency states are relatively less common and have normal blood levels. However, the functional activity of protein $\mathrm{C}$ is impaired. In general, the incidence of protein $\mathrm{C}$ deficiency is 1 in 200 to 500 [16]. Nedeltchev et al. reported $0.5 \%$ incidence rate of protein $\mathrm{C}$ deficiency in young IS patients [10]. An association between young IS and inherited protein $\mathrm{C}$ deficiency has been reported in individual case reports [17]. However, larger studies failed to demonstrate this relationship [18]. Protein C deficiency alone does not appear to increase the risk or arterial thrombosis, slightly higher incidence of myocardial infarction is noted when it is coupled with other vascular risk factors $[19,20]$. Uncommonly, some physiological and disease states like pregnancy, drugs, severe infection, and liver disease can produce an acquired protein $\mathrm{C}$ deficiency.

Protein $S$ acts as a cofactor for protein C. Isolated protein $\mathrm{S}$ deficiency may be seen in up to $10 \%$ patients with young IS. However, except for a few case reports, [21] significant relationship between this disorder and arterial thrombosis has never been established [18]. Instead, most of the patients with isolated protein S deficiency develop DVT, and they usually do so before the age of 25 years.

Antithrombin deficiency occurs in 1 of 5000 healthy blood donors [22]. Among patients with a first thrombotic event, the prevalence of this inherited disorder is approximately $0.5 \%$ to $1 \%[23,24]$. This protein may contribute to thrombotic events either by being deficient or dysfunctional. Again, direct association of antithrombin deficiency with arterial thrombosis remains controversial [22, 24].

\subsection{Disorders That Are Polygenic and Interact with Dietary or Environmental Factors. Cross-sectional and observational epidemiological studies have suggested that elevated plasma homocysteine concentrations might constitute a higher risk for major thrombotic events [25-27]. Some randomized trials demonstrated that homocysteine levels in the blood can be lowered by treatment with B vitamins and folic acid [28]. Homocysteine metabolism may be impaired due to genetic (abnormal enzymes in its metabolic pathway) as well as nutritional factors (vitamin B6, folate, and B12 deficiencies).}


The most common genetic mutation of hyperhomocysteinemia is a C-to-T substitution of nucleotide 677 (C677T) of the gene 5,10-methylenetetrahydrofolate reductase (MTHFR). The VITATOPS, a randomized, double-blind, placebocontrolled trial involving 8164 patients, recently tested the homocysteine-lowering multivitamin therapy in patients with transient ischemic attacks or strokes [29]. The study concluded that the daily administration of folic acid, vitamin B6, and vitamin B12 in patients with recent stroke or transient ischemic attack was safe but did not reduce the incidence of major vascular events as compared to the placebo.

\section{Acquired Thrombophilic Disorders}

4.1. Antiphospholipid Antibody Syndrome. The most common acquired thrombophilic disorder is antiphospholipid syndrome (APS). APS is a multisystem autoimmune disorder which can be either primary or secondary to other autoimmune diseases. It occurs more commonly in females and is characterized by arterial and venous thrombosis. It is associated with antiphospholipid antibodies such as lupus anticoagulant and anticardiolipin antibodies. A case control study suggested that lupus anticoagulant is a major risk factor for arterial thrombotic events in young women and found $17 \%$ of patients with IS as tested positive for the antibody [30]. Lupus anticoagulants are considered to carry a 5 to 16 times higher risk for thrombotic events than anticardiolipin antibodies [31].

4.2. Diagnosis of APS. APS can be diagnosed based on an international consensus criteria which was designed mainly for application to clinical studies of APS, and its diagnosis is based on clinical criteria of one or more occurrence of pregnancy morbidity or vascular thrombosis, and laboratory criteria of the presence of antiphospholipid antibodies on two or more occasions at least 12 weeks apart [32].

4.3. Laboratory Measurement of Antiphospholipid Antibodies. Consensus guidelines recommend screening for lupus anticoagulants (LA) with 2 or more phospholipid-dependent coagulation tests, which is best done when the patient is not receiving oral anticoagulants or unfractionated heparin, which can result in prolonged clotting times and subsequent false-positive results [33]. Both anticardiolipin antibodies and anti- $\beta_{2}$-glycoprotein I antibodies can be detected using enzyme-linked immunosorbent assay (ELISA) techniques, but many of these assays are not standardized. Consensus guidelines recommend semiquantitative reporting of anticardiolipin antibody results (low, medium, or high titer) [34].

4.4. Antithrombotic Treatment of APS for Patients with Arterial Thromboembolism. The choice of antithrombotic treatment is still controversial due to the lack of randomized treatment trials. Treatment choices should be individualized by balancing the risk of thrombosis and hemorrhage of each patient. Generally, the most common presentation of arterial thromboembolism in APS is ischemic stroke (13\%), and transient ischemic attack (7\%) of patients meeting consensus diagnostic criteria [35]. The presence of lupus anticoagulants or anticardiolipin antibodies have also been found to be associated with a 2-fold increase in first ischemic stroke [36].

The APASS study (APL and Stroke Study), [37] was a prospective cohort study within the Warfarin Aspirin Recurrent Stroke Study (WARSS), which was a randomized double-blind study comparing warfarin and aspirin for preventing recurrent stroke or death [38]. They found that patients treated with warfarin had a similar relative risk of recurrent ischemic stroke or death as patients treated with aspirin, and there was no difference whether patients were tested positive or negative for antiphosphospholipid antibodies [37]. Based on this, antithrombotic recommendations have been that both warfarin and aspirin are reasonable first choice antithrombotic treatments for patients presenting with a first ischemic stroke, with aspirin having the benefit of a lower bleeding risk and not requiring regular INR monitoring [39]. However, it should be noted that the median INR achieved in this study by the patients on warfarin was 1.9. There are currently no studies evaluating the optimal duration of treatment, and so patients are often treated indefinitely, similar to those patients with venous thromboembolism [39].

4.5. Heparin-Induced Thrombocytopenia. Heparin-induced thrombocytopenia (HIT) is another cause of transient acquired thrombophilia. It is due to heparin-dependent platelet activating immunoglobulin which further causes a temporary hypercoagulable state through several mechanisms. The risk of HIT-associated thrombosis ranges from $50 \%$ to $90 \%$, with higher risk of thrombosis in the lower platelet group [40]. Generally, venous thrombosis like deep vein thrombosis and pulmonary embolism are most common, but arterial thrombosis including lower limb ischemia, stroke, and myocardial infarction are also relatively common [40].

4.6. Lipoprotein (a) and Stroke. Another important cause of acquired thrombophilia is lipoprotein (a). It is a glycoprotein attached to the LDL. In a prospective study, lipoprotein (a) concentration was associated with chronic heart disease (risk ratio of $1.16,95 \% \mathrm{CI}, 1.11-1.22$ ) and ischemic stroke (risk ratio of 1.10, 95\%CI, 1.02-1.18) [41]. The mechanism is still unclear but increasing thrombosis and impaired fibrinolysis at the sites of plaque rupture are the possible causes [42].

4.7. Other Related Disorders That Increase the Risk of IS. Presence of thrombophilic state typically predisposes a patient predominantly to venous thrombosis and carries only a small risk for arterial thrombosis or IS. More typically, the thrombophilic disorders contribute to DVT in lower extremities and pelvic veins travels and an embolus from them may traverse through an atrial septal defect or patent foramen ovale (PFO) to cause paradoxical cerebral arterial embolization [43]. In a series with 125 young IS patients (age $34 \pm 7.3$ years), the PT G20210A variant and FV G1691A mutation were more frequent in the $\mathrm{PFO}$ positive group compared to the controls (PFO negative) [44]. However, the phenomenon of paradoxical embolization through a PFO remains largely 


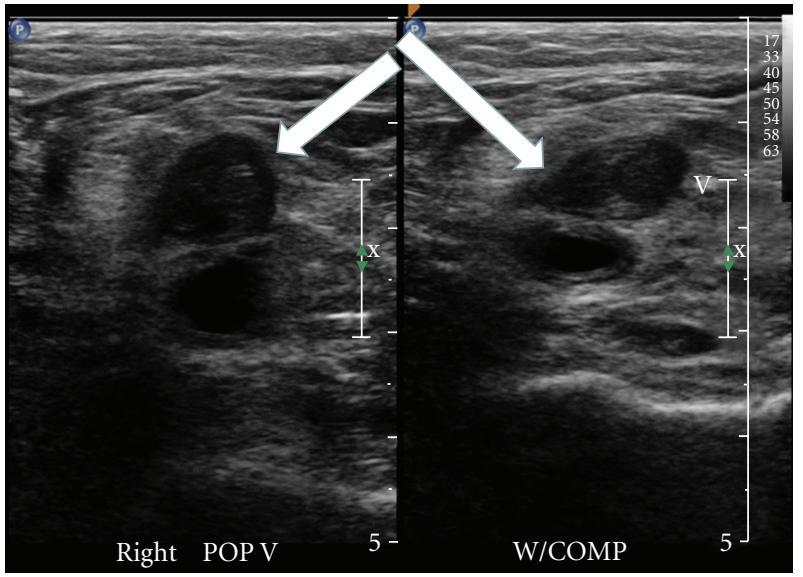

(a)

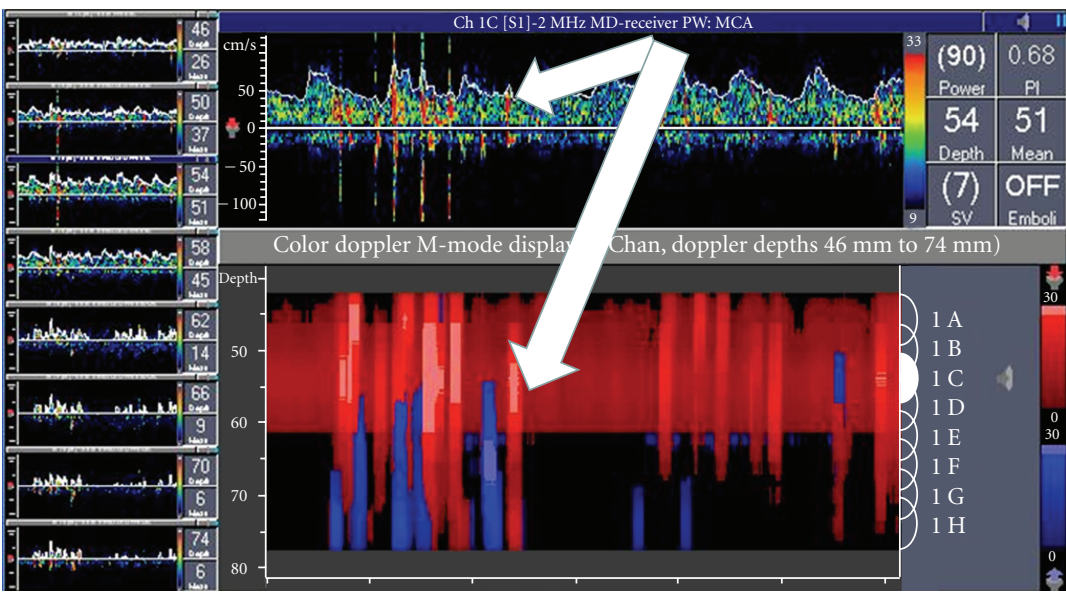

(c)

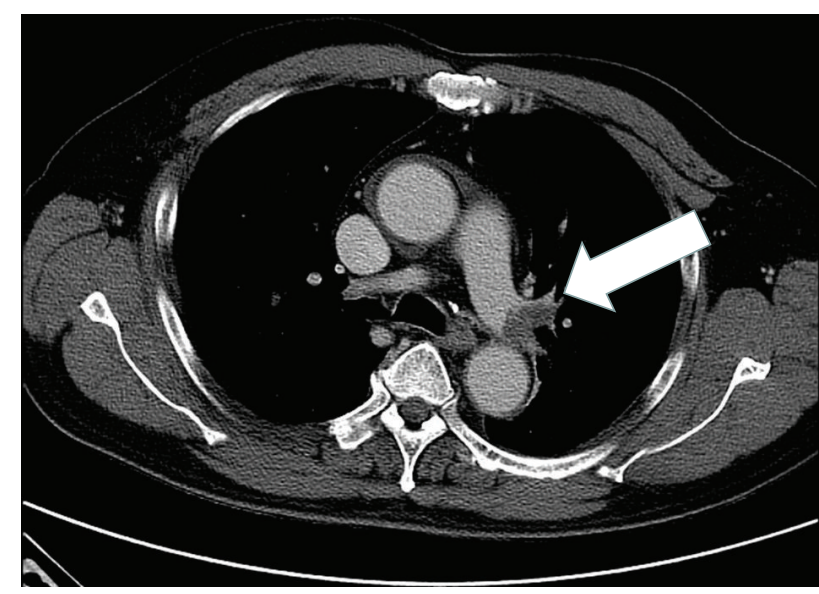

(b)

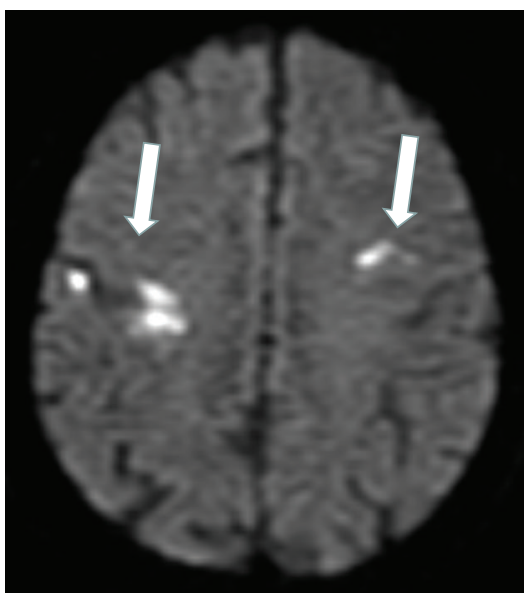

(d)

FIGURE 1: A 27-year-old woman, without any vascular risk factors, presented with mild right-sided weakness of suddenonset. She was noted to have swelling and pain in the right leg. Duplex sonography revealed extensive deep vein thrombosis involving the popliteal as well as superficial femoral vein, as characteristic noncompressibility and echogenic mass in the venous lumen. (a) Computerized tomographic angiography of thorax revealed filling defect in the left pulmonary artery. (b) Transcranial Doppler ultrasonographic monitoring of left middle cerebral artery during the "bubble test" (injection of agitated $9 \mathrm{~mL}$ normal saline and $1 \mathrm{~mL}$ air) revealed numerous microembolic signals, (c) suggestive of a right-to-left shunt (patent foramen ovale). Multiple acute ischemic infarcts involving many arterial territories were evident on the diffusion-weighted magnetic resonance imaging of the brain. (d) Blood tests were positive for lupus anticoagulant and anticardiolipin antibodies.

hypothetical. Various epidemiological studies have reported an increased incidence of PFO in cryptogenic stroke patients as compared to the general population. Since cerebral embolization through a PFO is considered to be the possible etiological mechanism, identification of the presence of a venous thrombus and its migration via the PFO are essential to confirm this pathogenic mechanism. However, both of these essential components are often difficult to establish due to various methodological and temporal issues [45]. Imaging findings in a patient with thrombophilia, extensive deep vein thrombosis, pulmonary embolism as well as multiple cerebral ischemic infarctions due to paradoxical embolization through a PFO are shown in Figure 1.

4.8. Synergisms. We believe that acute IS in young thrombophilic patients is the result of interaction between the underlying genetic disorder and environmental factors. This interaction might influence the stroke severity also. Common environmental factors that are believed to increase the risk of thrombotic events in patients with relevant genetic disorders include smoking, hypertension, dyslipidemia, and diabetes mellitus. In a study that looked at the cumulative effect of some of the inherited thrombophilias and their interactions with modifiable predisposing factors (smoking, hypertension), the odds for IS were 1.73 in subjects with one polymorphism. The risk increased to 3.00 if the subjects had two or more polymorphisms [46].

Oral contraceptive pills are believed to increase the risk of venous thrombotic events and, at times even IS, especially in patients with thrombophilia [47]. In a case-control study on women less than 45 years old with a first IS, oral contraceptives doubled the risk of IS in the first 6-18 months of use. 
The risk increased dramatically to 13 times higher in patients who were also carriers of Factor V Leiden mutation and 9 times higher with coexistent hyperhomocysteinemia [48].

\section{The Controversy of Testing for Thrombophilia}

Young IS patients, especially those without the common vascular risk factors and classified as "strokes of undetermined etiology" [2], are usually investigated for uncommon causes, with special emphasis on thrombophilic disorders. As a result, up to a third of all IS patients may undergo a barrage of tests for various thrombophilic states [49]. However, indiscriminate ordering of these tests is often debatable. The cost of this gamut of tests can run up to more than $\$ 1000$ US dollars in the United States [49], US \$500 in the United Kingdom, and US \$200 in Australia [50]. Apart from the high cost, these tests generate some psychological impact on the patients and their families, especially due to the possible genetic component [51]. Potential psychological and social consequences include fear, depression and worry [51], and difficulty of getting life or disability insurance in the future if the tests show an abnormal result [52].

The actual benefit of routine testing for thrombophilic disorders in IS remains uncertain $[49,50,53]$. The primary aims of testing for these uncommon disorders are to ascertain the definite diagnosis, possible prognostication, and to provide an appropriate treatment as well as secondary prophylaxis. Furthermore, primary prophylaxis may also be offered to family members of the proband who are carriers of a particular genetic defect but have not yet had a symptomatic thrombotic event [54]. However, the yield of performing extensive diagnostic tests is often poor and, in the majority of cases, no established curative measures exist.

\section{Who Should be Screened?}

The prevalence of thrombophilic disorders among the general population is low [7-10]. Furthermore, even when they occur with a higher prevalence (Factor V Leiden and prothrombin gene mutations in Caucasians), the thrombophilic states may not carry an alarmingly increased risk of thromboembolism [54]. The acquired thrombophilic disorders (lupus anticoagulant and anticardiolipin antibodies) confer a relatively higher risk for arterial thrombosis and IS [31]. However, the inherited thrombophilic disorders account for a very low thrombotic risk $[6,54,55]$. Furthermore, the prevalence of various thrombophilic disorders is very low in some ethnic groups of African or African-Caribbean descent $[6,54,55]$. Therefore, it has generally been believed that the routine screening of the general population is not justified.

Morris et al., in their analysis of the case-control studies of the 5 most commonly inherited thrombophilias with ischemic stroke: protein $\mathrm{C}$ and $\mathrm{S}$ deficiencies, antithrombin deficiency, factor $\mathrm{V}$ Leiden and prothrombin gene mutations, found no convincing associations with stroke, even in young patients and patients with patent foramen ovale [56]. In light of this, they have recommended that patients without a white ancestor should not be tested for Factor V Leiden or Prothrombin and that levels of protein $\mathrm{C}$ and protein $S$ should be interpreted cautiously in those of African descent. They also recommended that cryptogenic stroke patients with patent foramen ovale should be investigated for deep venous thrombosis in the legs and pelvic veins. As part of their recommendations, investigating for inherited thrombophilias should be done in patients having any of the other clinical features necessitating a thrombophilic workup as set out by WHO recommendations: history of venous thromboembolism-either unprovoked or in unusual location, family history of venous thromboembolism, thrombosis at a young age (less than 45 years old), and frequent thrombotic recurrences [57].

Walker et al. [6] observed a nonsignificant trend towards a higher prevalence of thrombophilia, especially in $20.5 \%$ of IS classified as "cardioembolic stroke" according to the TOAST classification [2]. The study postulated that the hypercoagulable state promoted the development of "red" fibrin thrombi in areas of stasis such as veins and heart chambers. These "red" clots were considered rich in fibrin and differed from the usual "white" clots, rich in platelet content, that develop in arteries [6]. Similarly, Carod-Artal et al. [58] found that prothrombotic conditions were more frequent among the young IS patients classified as "strokes of undetermined cause" [2]. However, they did not observe any association between inherited thrombophilic disorders and any of the pathogenic subtypes of IS among the older patients. Interestingly, isolated protein $\mathrm{S}$ deficiency was associated with "stroke of undetermined cause" in the young patients.

Selection of patients for performing various diagnostic tests for the thrombophilc disorders should not be influenced by IS involving a specific vascular territory. For example, anterior cerebral artery (ACA) territory infarctions, comprising less than $3 \%$ of IS, are usually considered cardioembolic in origin [59]. Stroke in ACA territory are, therefore, often investigated for an underlying cardioembolic or thrombophilic cause. However, in some ethnic populations, especially Chinese, ACA may be involved in the generalized atherosclerotic process [60]. IS resulting from the thrombophilic disorders might involve any arterial territory and often affects multiple arterial territories together [61]. The more useful and practical approach of ordering various diagnostic tests for the uncommon thrombophilic states tests should be determined by a detailed clinical history, physical examination, imaging studies and evaluating whether an underlying hypercoagulable state appears more likely. History of a prior thromboembolic event, especially that occurred after minimal stimuli or at unusual sites is an important clue for thrombophilic disorders [62]. Similarly, venous thrombosis is the most common manifestation of coagulopathy [63] while a history of unexplained miscarriage may signify the presence of antiphospholipid syndrome [64]. Some other clues that might suggest the presence of a thrombophilic disorder include thrombocytopenia, livedo reticularis, or Sneddon syndrome (skin necrosis during initiation of oral anticoagulant therapy), family history of clotting events at a young age, absence of conventional 
vascular risk factors, presence of malignancy or sepsis, and recurrent thrombotic events despite appropriate treatment [65]. History of previous venous thrombosis and miscarriage is the most important fact that is often missing in patients' medical records which leads to a delay in diagnosis [49].

An important factor that should be used in selecting patient for testing the thrombophilic disorders is age. While thrombophilia is rarely associated with arterial occlusive disease in adults, it constitutes an important cause in childhood and young IS. Accordingly, Duran et al. found that $57 \%$ of pediatric IS patients tested positive for at least one thrombophilic marker as compared to $15 \%$ of age-matched controls [66]. Furthermore, $10 \%$ of the pediatric population had 2 or more positive markers. Similarly, a high prevalence of antiphospholipid syndrome is observed in children with idiopathic cerebral ischemia [67], with $76 \%$ of children aged 5 to 16 years tested positive for lupus anticoagulant or anticardiolipin antibodies. However, it should always be kept in mind that atherosclerosis and cardioembolism still constitute more likely causes [68] as compared to the thrombophilic disorders that account for only $10 \%$ to $15 \%$ of the cases of IS in the young [3]. In another study of subjects less than 18 years of age, half to two thirds of IS demonstrated thrombophilic states [69]. Considering the rarity of childhood IS, congenital heart disease would have complemented multiple coexisting thrombophilic states in causing the high prevalence of IS [69]. Finally, screening for prothrombotic states should also be considered in symptomatic siblings and first-degree family members to identify patients at relatively highrisk [70].

\section{What Should be Screened?}

Some young patients suffer from multiple thrombophilic disorders that confer an additive or multiplicative risk of thromboembolism. For example, the combined defects of protein $\mathrm{C}$ or protein $\mathrm{S}$ deficiencies plus Factor V Leiden, or the acquirement of hyperhomocysteinemia with Factor V Leiden, increase the risk of thromboembolism compared to either defect in isolation. Similarly, a combined antithrombin III deficiency with Factor V Leiden mutation poses a very high risk of thromboembolism when compared to either defect alone. Therefore, the laboratory screening should be comprehensive and avoid missing the coexisting defect [54]. Accordingly, functional assays for proteins $\mathrm{C}$, protein $\mathrm{S}$, and antithrombin III should be performed if activated protein C (APC) resistance is observed; prothrombin 20210A, fibrinogen, homocysteine, D-dimers, and factor VIII should be tested carefully if there is coexisting ulcerative colitis [71]. It is important that a diagnostic search protocol should include tests for both inherited and acquired thrombophilic disorders. Finally, assessment of plasma homocysteine has been advised in all IS patients since it is considered easily reversible with vitamin supplementation [71]. However, the recently published VITATOPS trial failed to demonstrate any significant beneficial effects of this approach [29].

\section{What Is the Appropriate Timing for Screening?}

Since the therapeutic approach (anticoagulation and thrombolytic therapy) determines the clinical outcomes, early diagnosis of the thrombophilic disorders plays an important role. Furthermore, the timing of test performance of some of the thrombophilic defects (like protein C, protein S, antithrombin III and fibrinogen levels) is often critical since these proteins can behave as acute phase reactants and erroneously elevated levels of these factors may be observed in patients with acute thrombotic events [54, 71]. On the other hand, the plasma levels of vitamin K-dependent proteins (protein $\mathrm{C}$, protein $\mathrm{S}$ and APC resitance) may not be reliable in patients taking vitamin $\mathrm{K}$ antagonists [72]. Similarly, antithrombin III measurements are unreliable in patients receiving heparin. Therefore, it is suggested that plasma-based assays for these disorders should be repeated 3 to 6 months after the initial thrombotic episode to avoid false-positive results and avoid unnecessary prolonged anticoagulation therapy. The assays for these disorders are recommended after discontinuation of oral anticoagulant treatment or heparin for at least 2 weeks $[54,71]$.

DNA-based assays are not affected by acute thrombotic events or use of anticoagulation and thrombolytic therapy. Therefore, screening for these genetic mutations can be performed reliably at any time following a thrombotic event $[54,71]$.

Finally, natural history for some of the thrombophilic disorders should be considered during the interpretation of their diagnostic assays. Accordingly, lipoprotein (a) levels increase during the first year of birth. Hence, it is necessary to repeat testing 8 to 12 months after the acute thrombotic onset in neonates suffering from thromboembolism [71].

\section{Factors to be Considered during the Interpretation of Diagnostic Tests}

As mentioned above, the interpretation of the tests for some of the thrombophilia markers depends on its timing in relation to the acute thrombotic event as well as concurrent medications, especially vitamin $\mathrm{K}$ antagonists and heparin. In addition, patients' age also might affect the results of some these markers. For example, antithrombin III levels decline with increasing age, and the reference range may therefore need some adjustments accordingly [6]. Another important factor that may alter the results is patients' ethnicity. The conventionally employed reference ranges for some biomarkers of thrombophilia may lead to false positive diagnoses in black Africans or black Caribbeans due to their inherent lower concentrations when compared to the Caucasian population [14].

The presence of concomitant medical conditions and drug therapies should also be taken into consideration during the interpretation of results. For example, antithrombin III levels can be relatively lower in liver disease, nephritic syndrome, inflammatory bowel diseases and pregnancy; liver disease can be associated with lowered protein $\mathrm{C}$ and $\mathrm{S}$ levels; pregnancy can result in lowered protein $S$ levels and acquired 
APC-resistance. Furthermore, protein $S$ levels and the acquired APC resistance may be affected by sex-hormonal treatment in females [72]. Correct interpretation of anticardiolipin antibody titers can also be difficult in some patients, as the blood levels can be affected by other conditions such as viral, bacterial or parasitic infections, lymphoproliferative disorders, paraproteinemia, or drugs such as phenothiazine, procainamide, phenytoin, quinidine and hydralazine [73]. Therefore, other clinical or laboratory features of APS should be sought for an accurate diagnosis [43].

\section{What Secondary Stroke Prevention Method Should be Instituted If the Screening Is Positive?}

In the event that the screening was done indiscriminately for a stroke patient, it is reasonable to first interpret the results with caution. One must first evaluate whether a more obvious stroke mechanism is present, or whether a coexisting condition is already present which would already necessitates the use of anticoagulation, for example a cardioembolic source. The results should also be assessed if they are truly positive, for example if the protein $\mathrm{C}$ or $\mathrm{S}$ levels were drawn shortly after an acute thrombosis, or if the blood test were done with patient still on anticoagulant therapy. Lastly, one should interpret the significance of the positive result in light of the patient's cardiovascular risk profile and age, for example, it would have greater implications in a young patient without cardiovascular risk factors [74].

Treatment of an ischemic stroke is not as clearly defined as that for venous thromboembolism in all of the inherited thrombophilias. Acute venous thromboembolism is treated with heparin or low-molecular-weight heparin and then warfarin to achieve a target INR of 2.0 to 3.0 for at least 6 months to an indefinite period of time [57]. For ischemic stroke, however, there is only 1 prospective observational study comparing cryptogenic stroke patients with and without thrombophilia, and the use of anticoagulation did not affect outcomes [75].

Oral anticoagulation is therefore not recommended for asymptomatic carriers of these defects, though symptomatic patients may possibly be treated in the same way as that of venous thromboembolism, though anticoagulation may be avoided in the acute phase due to the concerns for hemorrhagic transformation of the infarction [56].

\section{Conclusion}

Our understanding of various mechanisms for thromboembolism has improved considerably in the past decade. Furthermore, the diagnostic tests have become simpler, cheaper, faster, more reliable, and widely available. Low prevalence and diagnostic yield of various thrombophilic disorders does not support strongly the routine screening of the general population. However, a careful selection of high-risk patients, the timing of testing, and the type of tests often contribute towards appropriate therapeutic decision making [76]. Future research in this field would improve our understanding about various thrombophilic disorders and aid in developing optimal therapeutic and preventive strategies for younger ischemic stroke patients.

\section{References}

[1] J. F. Varona, J. M. Guerra, F. Bermejo, J. A. Molina, and A. Gomez de la Cámara, "Causes of ischemic stroke in young adults, and evolution of the etiological diagnosis over the long term," European Neurology, vol. 57, no. 4, pp. 212-218, 2007.

[2] H. P. Adams Jr., B. H. Bendixen, L. J. Kappelle et al., "Classification of subtype of acute ischemic stroke: definitions for use in a multicenter clinical trial. TOAST. Trial of Org 10172 in Acute Stroke Treatment," Stroke, vol. 24, no. 1, pp. 35-41, 1993.

[3] S. J. Kittner, B. J. Stern, M. Wozniak et al., "Cerebral infarction in young adults: the Baltimore-Washington Cooperative Young Stroke Study," Neurology, vol. 50, no. 4, pp. 890-894, 1998.

[4] J. Putaala, A. J. Metso, T. M. Metso et al., "Analysis of 1008 consecutive patients aged 15 to 49 with first-ever ischemic stroke the Helsinki young stroke registry," Stroke, vol. 40, no. 4, pp. 1195-1203, 2009.

[5] E. S. Roach, M. R. Golomb, R. Adams et al., "Management of stroke in infants and children: a scientific statement from a special writing group of the american heart association stroke council and the council on cardiovascular disease in the young," Stroke, vol. 39, no. 9, pp. 2644-2691, 2008.

[6] I. D. Walker, M. Greaves, and F. E. Preston, "Investigation and management of heritable thrombophilia," British Journal of Haematology, vol. 114, no. 3, pp. 512-528, 2001.

[7] G. J. Hankey, J. W. Eikelboom, F. M. van Bockxmeer, E. Lofthouse, N. Staples, and R. I. Baker, "Inherited thrombophilia in ischemic stroke and its pathogenic subtypes," Stroke, vol. 32, no. 8, pp. 1793-1799, 2001.

[8] I. Martinelli, P. M. Mannucci, V. de Stefano et al., "Different risks of thrombosis in four coagulation defects associated with inherited thrombophilia: a study of 150 families," Blood, vol. 92, no. 7, pp. 2353-2358, 1998.

[9] P. M. Ridker, C. H. Hennekens, K. Lindpaintner, M. J. Stampfer, P. R. Eisenberg, and J. P. Miletich, "Mutation in the gene coding for coagulation factor $\mathrm{v}$ and the risk of myocardial infarction, stroke, and venous thrombosis in apparently healthy men," New England Journal of Medicine, vol. 332, no. 14, pp. 912-917, 1995.

[10] K. Nedeltchev, T. A. der Maur, D. Georgiadis et al., "Ischaemic stroke in young adults: predictors of outcome and recurrence," Journal of Neurology, Neurosurgery and Psychiatry, vol. 76, no. 2, pp. 191-195, 2005.

[11] F. R. Rosendaal, C. J. M. Doggen, A. Zivelin et al., "Geographic distribution of the 20210 G to A prothrombin variant," Thrombosis and Haemostasis, vol. 79, no. 4, pp. 706-708, 1998.

[12] V. de Stefano, P. Chiusolo, K. Paciaroni et al., "Prothrombin G20210A mutant genotype is a risk factor for cerebrovascular ischemic disease in young patients," Blood, vol. 91, no. 10, pp. 3562-3565, 1998.

[13] K. H. Reuner, A. Ruf, A. Grau et al., "Prothrombin gene G20210- $\dot{\text { A }}$ transition is a risk factor for cerebral venous thrombosis," Stroke, vol. 29, no. 9, pp. 1765-1769, 1998.

[14] P. Jerrard-Dunne, A. Evans, R. McGovern et al., "Ethnic differences in markers of thrombophilia implications for the 
investigation of ischemic stroke in multiethnic populations: the south London ethnicity and stroke study," Stroke, vol. 34, no. 8, pp. 1821-1826, 2003.

[15] S. A. Mayer, R. L. Sacco, A. Hurlet-Jensen, T. Shi, and J. P. Mohr, "Free protein $S$ deficiency in acute ischemic stroke: a case-control study," Stroke, vol. 24, no. 2, pp. 224-227, 1993.

[16] R. C. Tait, I. D. Walker, P. H. Reitsma et al., "Prevalence of protein $\mathrm{C}$ deficiency in the healthy population," Thrombosis and Haemostasis, vol. 73, no. 1, pp. 87-93, 1995.

[17] J. Kohler, J. Kasper, I. Witt, and G. M. von Reutern, "Ischemic stroke due to protein C deficiency," Stroke, vol. 21, no. 7, pp. 1077-1080, 1990.

[18] X. Douay, C. Lucas, C. Caron, J. Goudemand, and D. Leys, "Antithrombin, protein $\mathrm{C}$ and protein S levels in 127 consecutive young adults with ischemic stroke," Acta Neurologica Scandinavica, vol. 98, no. 2, pp. 124-127, 1998.

[19] I. Y. Tiong, M. L. Alkotob, and S. Ghaffari, "Protein C deficiency manifesting as an acute myocardial infarction and ischaemic stroke," Heart, vol. 89, no. 2, p. E7, 2003.

[20] M. Camerlingo, G. Finazzi, L. Casto, C. Laffranchi, T. Barbui, and A. Mamoli, "Inherited protein C deficiency and nonhemorrhagic arterial stroke in young adults," Neurology, vol. 41, no. 9, pp. 1371-1373, 1991.

[21] A. Girolami, P. Simioni, A. R. Lazzaro, and I. Cordiano, "Severe arterial cerebral thrombosis in a patient with protein $S$ deficiency (moderately reduced total and markedly reduced free protein S): a family study," Thrombosis and Haemostasis, vol. 61, no. 1, pp. 144-147, 1989.

[22] R. C. Tait, I. D. Walker, D. J. Perry et al., "Prevalence of antithrombin deficiency in the healthy population," British Journal of Haematology, vol. 87, no. 1, pp. 106-112, 1994.

[23] J. Mateo, A. Oliver, M. Borrell, N. Sala, and J. Fontcuberta, "Laboratory evaluation and clinical characteristics of 2,132 consecutive unselected patients with venous thromboembolism-results of the Spanish multicentric study on thrombophilia (EMET-Study)," Thrombosis and Haemostasis, vol. 77, no. 3, pp. 444-451, 1997.

[24] D. A. Lane, P. M. Mannucci, K. A. Bauer et al., "Inherited thrombophilia: part 1," Thrombosis and Haemostasis, vol. 76, no. 5, pp. 651-662, 1996.

[25] J. W. Eikelboom, G. J. Hankey, S. S. Anand, E. Lofthouse, N. Staples, and R. I. Baker, "Association between high homocyst(e)ine and ischemic stroke due to large- and smallartery disease but not other etiologic subtypes of ischemic stroke," Stroke, vol. 31, no. 5, pp. 1069-1075, 2000.

[26] J. P. Casas, L. E. Bautista, L. Smeeth, P. Sharma, and A. D. Hingorani, "Homocysteine and stroke: evidence on a causal link from mendelian randomisation," Lancet, vol. 365, no. 9455, pp. 224-232, 2005.

[27] D. S. Wald, N. J. Wald, J. K. Morris, and M. Law, "Folic acid, homocysteine, and cardiovascular disease: judging causality in the face of inconclusive trial evidence," British Medical Journal, vol. 333, no. 7578, pp. 1114-1117, 2006.

[28] Homocysteine Lowering Trialists' Collaboration, "Lowering blood homocysteine with folic acid based supplements: metaanalysis of randomised trials," British Medical Journal, vol. 316, no. 7135, pp. 894-898, 1998.

[29] The VITATOPS Trial Study Group, "B vitamins in patients with recent transient ischaemic attack or stroke in the VITAmins TO Prevent Stroke (VITATOPS) trial: a randomised, double-blind, parallel, placebo-controlled trial," Lancet Neurology, vol. 9, no. 9, pp. 855-865, 2010.
[30] R. T. Urbanus, B. Siegerink, M. Roest, F. R. Rosendaal, P. G. de Groot, and A. Algra, "Antiphospholipid antibodies and risk of myocardial infarction and ischaemic stroke in young women in the RATIO study: a case-control study," Lancet Neurology, vol. 8, no. 11, pp. 998-1005, 2009.

[31] M. Galli, D. Luciani, G. Bertolini, and T. Barbui, "Lupus anticoagulants are stronger risk factors for thrombosis than anticardiolipin antibodies in the antiphospholipid syndrome: a systematic review of the literature," Blood, vol. 101, no. 5, pp. 1827-1832, 2003.

[32] S. Miyakis, M. D. Lockshin, T. Atsumi et al., "International consensus statement on an update of the classification criteria for definite antiphospholipid syndrome (APS)," Journal of Thrombosis and Haemostasis, vol. 4, no. 2, pp. 295-306, 2006.

[33] W. Lim, M. A. Crowther, and J. W. Eikelboom, "Management of antiphospholipid antibody syndrome: a systematic review," Journal of the American Medical Association, vol. 295, no. 9, pp. 1050-1057, 2006.

[34] E. N. Harris, "Special report. The second international anti-cardiolipin standardization workshop/the Kingston antiphospholipid antibody study (KAPS) group," American Journal of Clinical Pathology, vol. 94, no. 4, pp. 476-484, 1990.

[35] R. Cervera, J. C. Piette, J. Font et al., "Antiphospholipid syndrome: clinical and immunologic manifestations and patterns of disease expression in a cohort of 1,000 patients," Arthritis and Rheumatism, vol. 46, no. 4, pp. 1019-1027, 2002.

[36] R. L. Brey, C. L. Stallworth, D. L. McGlasson et al., "Antiphospholipid antibodies and stroke in young women," Stroke, vol. 33, no. 10, pp. 2396-2400, 2002.

[37] S. R. Levine, R. L. Brey, B. C. Tilley et al., "Antiphospholipid antibodies and subsequent thrombo-occlusive events in patients with ischemic stroke," Journal of the American Medical Association, vol. 291, no. 5, pp. 576-584, 2004.

[38] J. P. Mohr, J. L. P. Thompson, R. M. Lazar et al., "A comparison of warfarin and aspirin for the prevention of recurrent ischemic stroke," New England Journal of Medicine, vol. 345, no. 20, pp. 1444-1451, 2001.

[39] W. Lim, “Antiphospholipid antibody syndrome," Hematology, pp. 233-239, 2009.

[40] T. E. Warkentin, "Clinical picture of heparin-induced thrombocytopenia," in Heparin-Induced Thrombocytopenia, T. E. Warkentin and A. des Greinacher, Eds., pp. 43-86, Marcel Dekker, New York, NY, USA, 2nd edition, 2001.

[41] S. Erqou, S. Kaptoge, P. L. Perry et al., "Lipoprotein(a) concentration and the risk of coronary heart disease, stroke, and nonvascular mortality," Journal of the American Medical Association, vol. 302, no. 4, pp. 412-423, 2009.

[42] J. D. Spence, "The role of lipoprotein(a) in the formation of arterial plaques, stenoses and occlusions," Canadian Journal of Cardiology, vol. 26, pp. 37A-40A, 2010.

[43] S. R. Levine, "Hypercoagulable states and stroke: a selective review," CNS Spectrums, vol. 10, no. 7, pp. 567-578, 2005.

[44] A. Pezzini, E. del Zotto, M. Magoni et al., "Inherited thrombophilic disorders in young adults with ischemic stroke and patent foramen ovale," Stroke, vol. 34, no. 1, pp. 28-33, 2003.

[45] V. K. Sharma, H. L. Teoh, and B. P. L. Chan, "Patent foramen ovale and prothrombotic markers in young stroke patients," Blood Coagulation and Fibrinolysis, vol. 19, no. 4, pp. 326-327, 2008.

[46] A. Pezzini, M. Grassi, E. del Zotto et al., "Cumulative effect of predisposing genotypes and their interaction with modifiable factors on the risk of ischemic stroke in young adults," Stroke, vol. 36, no. 3, pp. 533-539, 2005. 
[47] A. J. C. Slooter, F. R. Rosendaal, B. C. Tanis, J. M. Kemmeren, Y. van der Graaf, and A. Algra, "Prothrombotic conditions, oral contraceptives, and the risk of ischemic stroke," Journal of Thrombosis and Haemostasis, vol. 3, no. 6, pp. 1213-1217, 2005.

[48] I. Martinelli, T. Battaglioli, I. Burgo, S. Di Domenico, and P. M. Mannucci, "Oral contraceptive use, thrombophilia and their interaction in young women with ischemic stroke," Haematologica, vol. 91, no. 6, pp. 844-847, 2006.

[49] C. Bushnell, Z. Siddiqi, J. C. Morgenlander, and L. B. Goldstein, "Use of specialized coagulation testing in the evaluation of patients with acute ischemic stroke," Neurology, vol. 56, no. 5, pp. 624-627, 2001.

[50] G. J. Hankey and J. W. Eikelboom, "Routine thrombophilia testing in stroke patients is unjustified," Stroke, vol. 34 , no. 8 , pp. 1826-1827, 2003.

[51] D. M. Cohn, F. Vansenne, A. A. Kaptein, C. A. J. M. de Borgie, and S. Middeldorp, "The psychological impact of testing for thrombophilia: a systematic review," Journal of Thrombosis and Haemostasis, vol. 6, no. 7, pp. 1099-1104, 2008.

[52] S. J. M. Homsma, R. Huijgen, S. Middeldorp, E. J. G. Sijbrands, and J. J. P. Kastelein, "Molecular screening for familial hypercholesterolaemia: consequences for life and disability insurance," European Journal of Human Genetics, vol. 16, no. 1, pp. 14-17, 2008.

[53] D. M. Cohn, S. Roshani, and S. Middeldorp, "Thrombophilia and venous thromboembolism: implications for testing," Seminars in Thrombosis and Hemostasis, vol. 33, no. 6, pp. 573581, 2007.

[54] A. Tripodi and P. M. Mannucci, "Laboratory investigation of thrombophilia," Clinical Chemistry, vol. 47, no. 9, pp. 15971606, 2001.

[55] C. D. Bushnell and L. B. Goldstein, "Diagnostic testing for coagulopathies in patients with ischemic stroke," Stroke, vol. 31, no. 12, pp. 3067-3078, 2000.

[56] J. G. Morris, S. Singh, and M. Fisher, "Testing for inherited thrombophilias in arterial stroke: can it cause more harm than good?" Stroke, vol. 41, no. 12, pp. 2985-2990, 2010.

[57] V. Boulyjenkov, "Inherited thrombophilia: memorandum from a joint $\mathrm{WHO}$ /international society on Thrombosis and Haemostasis meeting," Bulletin of the World Health Organization, vol. 75, no. 3, pp. 177-189, 1997.

[58] F. J. Carod-Artal, S. V. Nunes, D. Portugal, T. V. Fernandes Silva, and A. P. Vargas, "Ischemic stroke subtypes and thrombophilia in young and elderly Brazilian stroke patients admitted to a rehabilitation hospital," Stroke, vol. 36, no. 9, pp. 2012-2014, 2005.

[59] G. Gacs, A. J. Fox, H. J. M. Barnett, and F. Vinuela, "Occurrence and mechanisms of occlusion of the anterior cerebral artery," Stroke, vol. 14, no. 6, pp. 952-959, 1983.

[60] P. K. Loh and V. K. Sharma, "Atherosclerosis in congenital azygous solitary A2 anterior cerebral artery with simultaneous bifrontal infarctions," Journal of Neurology, Neurosurgery and Psychiatry, vol. 81, no. 2, article 203, 2010.

[61] S. R. Levine, M. J. Deegan, N. Futrell, and K. M. A. Welch, "Cerebrovascular and neurologic disease associated with antiphospholipid antibodies: 48 cases," Neurology, vol. 40, no. 8, pp. 1181-1189, 1990.

[62] M. Amiri, J. W. Schmidley, L. M. Fink, and S. M. Nazarian, "Is testing for inherited coagulation inhibitor deficiencies in young stroke patients worthwhile?" Clinical Neurology and Neurosurgery, vol. 102, no. 4, pp. 219-222, 2000.
[63] M. L. Brigden, "The hypercoagulable state: who, how, and when to test and treat," Postgraduate Medicine, vol. 101, no. 5, pp. 249-263, 1997.

[64] M. D. Lockshin, L. R. Sammaritano, and S. Schwartzman, "Validation of the Sapporo criteria for antiphospholipid syndrome," Arthritis and Rheumatism, vol. 43, no. 2, pp. 440443, 2000.

[65] M. C. Fields and S. R. Levine, "Thrombophilias and stroke: diagnosis, treatment, and prognosis," Journal of Thrombosis and Thrombolysis, vol. 20, no. 2, pp. 113-126, 2005.

[66] R. Duran, B. Biner, M. Demir, C. Celtik, and S. Karasalihoğlu, "Factor V Leiden mutation and other thrombophilia markers in childhood ischemic stroke," Clinical and Applied Thrombosis/Hemostasis, vol. 11, no. 1, pp. 83-88, 2005.

[67] L. Angelini, A. Ravelli, R. Caporali, and A. Martini, "Antiphospholipid antibodies in children with idiopathic cerebral ischaemia," Lancet, vol. 344, no. 8931, article 1232, 1994.

[68] R. G. Hart and V. T. Miller, "Cerebral infarction in young adults: a practical approach,” Stroke, vol. 14, no. 1, pp. 110114, 1983.

[69] C. Amlie-Lefond, G. Sébire, and H. J. Fullerton, "Recent developments in childhood arterial ischaemic stroke," Lancet Neurology, vol. 7, no. 5, pp. 425-435, 2008.

[70] D. Green, "Thrombophilia and stroke," Topics in Stroke Rehabilitation, vol. 10, no. 3, pp. 21-33, 2003.

[71] B. R. Brenner, U. Nowak-Göttl, A. Kosch, M. Manco-Johnson, and M. Laposata, "Diagnostic studies for thrombophilia in women on hormonal therapy and during pregnancy, and in children," Archives of Pathology and Laboratory Medicine, vol. 126, no. 11, pp. 1296-1303, 2002.

[72] V. de Stefano, E. Rossi, K. Paciaroni, and G. Leone, "Screening for inherited thrombophilia: indications and therapeutic implications," Haematologica, vol. 87, no. 10, pp. 1095-1108, 2002.

[73] M. Greaves, H. Cohen, S. J. Machin, and I. Mackie, "Guidelines on the investigation and management of the antiphospholipid syndrome," British Journal of Haematology, vol. 109, no. 4, pp. 704-715, 2000.

[74] B. L. Cucchiara, "Evaluation and management of stroke," Hematology, pp. 293-301, 2009.

[75] R. Weber, M. Goertler, J. Benemann, H. C. Diener, and C. Weimar, "Prognosis after cryptogenic cerebral ischemia in patients with coagulopathies," Cerebrovascular Diseases, vol. 28, no. 6, pp. 611-617, 2009.

[76] J. R. Payne and B. Coull, "Antithrombotic therapy for stroke in young adults," Journal of Thrombosis and Thrombolysis, vol. 20, no. 2, pp. 127-132, 2005. 


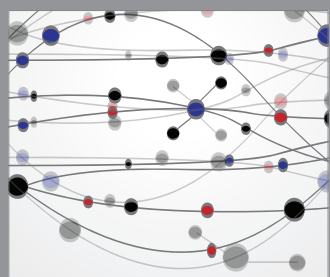

The Scientific World Journal
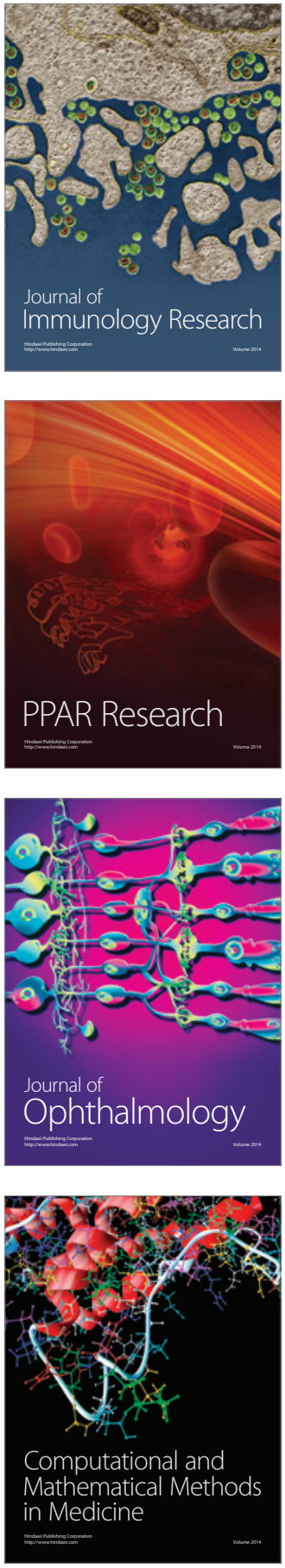

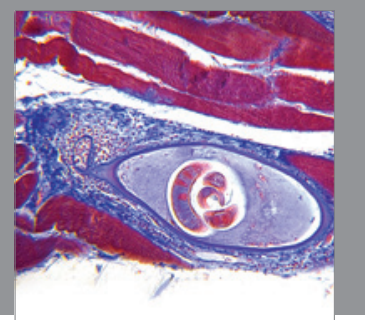

Gastroenterology

Research and Practice
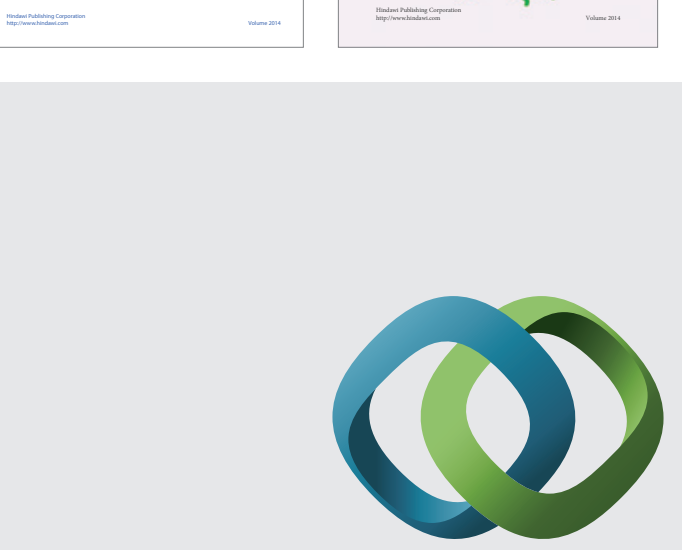

\section{Hindawi}

Submit your manuscripts at

http://www.hindawi.com
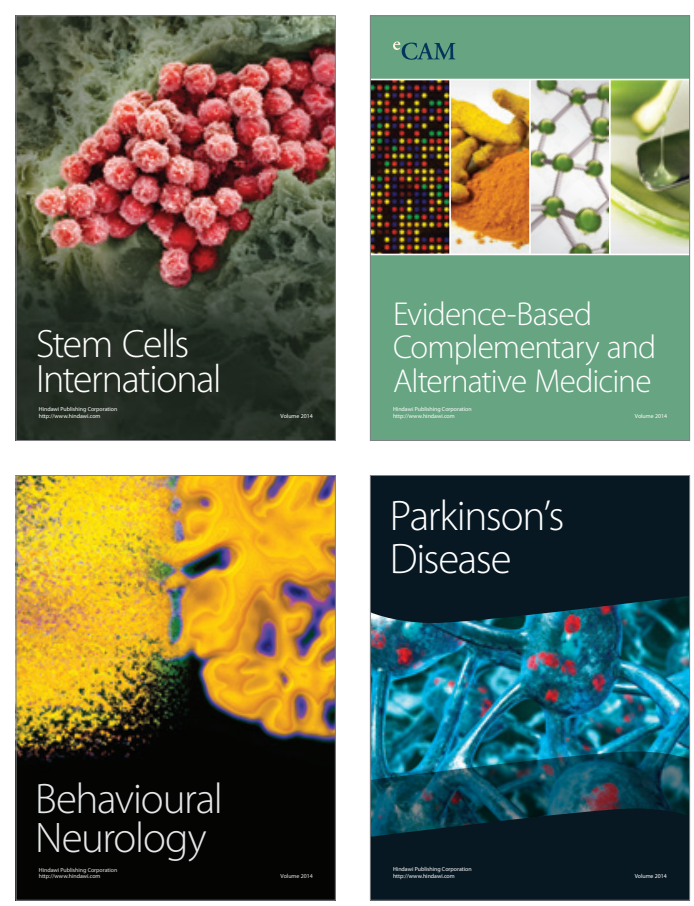

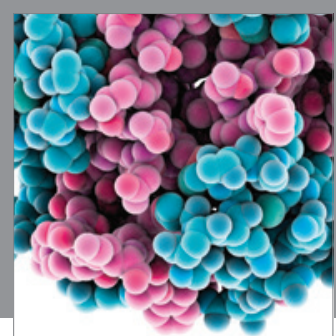

Journal of
Diabetes Research

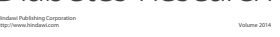

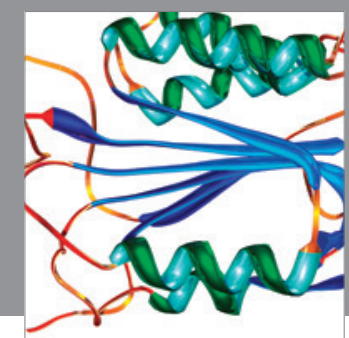

Disease Markers
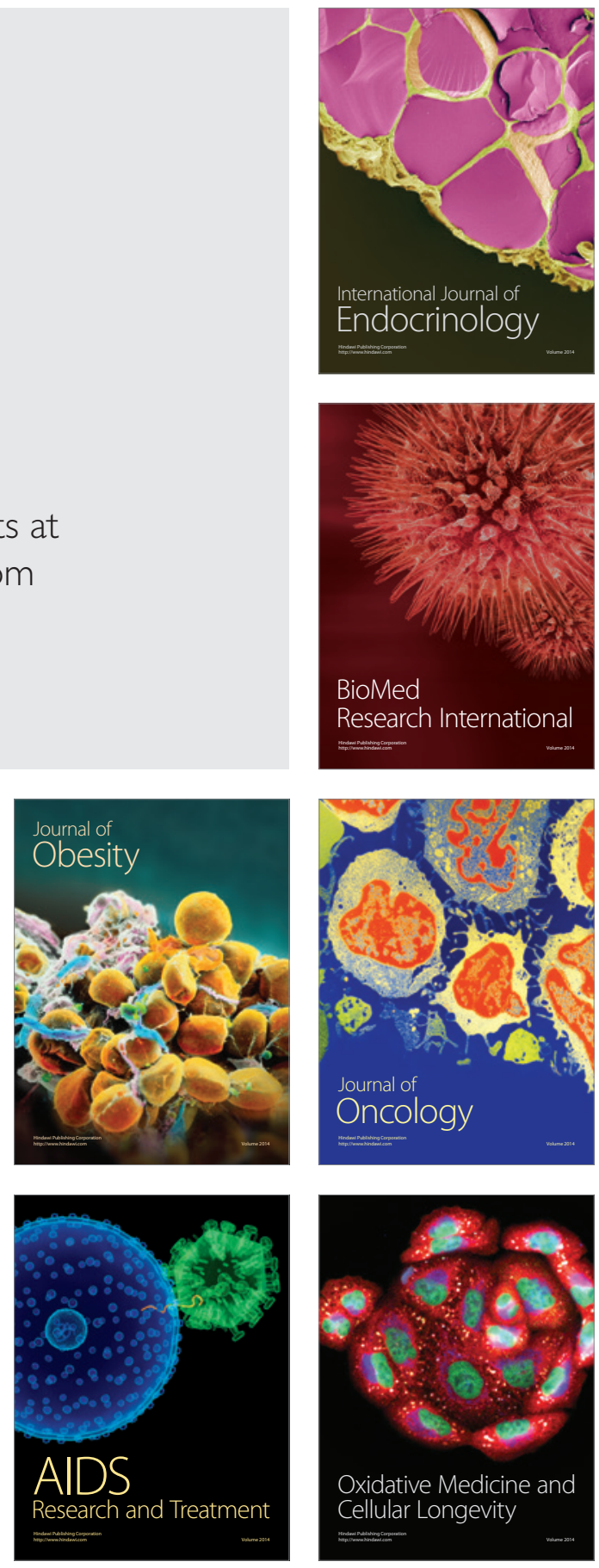